IAU Colloquium 190 on Magnetic Cataclysmic Variables

ASP Conference Series, Vol. 315, 2004

Sonja Vrielmann 8 Mark Cropper, eds.

\title{
Multi-Epoch spectroscopy and XMM-Newton observations of RX J2115-58
}

\author{
Mark Cropper, Gavin Ramsay \\ Mullard Space Science Laboratory, University College London, \\ Holmbury St Mary, Dorking, Surrey RH5 6NT, United Kingdom \\ Tom Marsh \\ Department of Physics and Astronomy, University of Southampton \\ Hampshire SO17 1BJ, United Kingdom
}

\begin{abstract}
We present here phase-resolved optical spectroscopy and Xray light curves of the asynchronous polar RX J2115-58 as they change on the beat period.
\end{abstract}

\section{Introduction}

RX J2115-58 was discovered as part of the ROSAT all-sky survey and independently by EUVE. Further observations by Schwope et al (1997) and Ramsay et al (1999) showed that it was an asynchronous polar with a beat period of 6.3 days. Such a short period allows us to study the effect of a changing orientation between the magnetic field and accretion stream on an easily observable timescale.

\section{AAT Spectroscopy}

The left plot in Fig. 1 shows phase-folded line profiles of $\mathrm{H} \beta$ (top), and HeII 4686 (bottom) on the nights of 2000 July 20,22 and 24 , evenly sampling the beat cycle (left to right). These were taken using the AAT RGO spectrograph. The profiles are complex, with significant changes evident over the beat cycle. An example is the enhanced heating of the secondary on the first night, evident in the strong narrow profile in the $\mathrm{H} \beta$ line (but not the HeII 4686), and the enhancement at $\phi \sim 0$ on July 22 and 24 , which is manifested in a brightening of the ballistic stream in the Doppler tomograms at these beat phases.

\section{XMM-Newton Observations}

The plot on the right of Fig. 1 shows light curves in hard X-rays from our $X M M$ Newton observations of this system (we also have UV light curves from the $X M M$ Optical Monitor). They consist of 7 separate observations of two orbital periods, one each day, evenly sampling the beat period. They are extremely variable from night to night, but these in the hard band are not dissimilar to those seen in the 

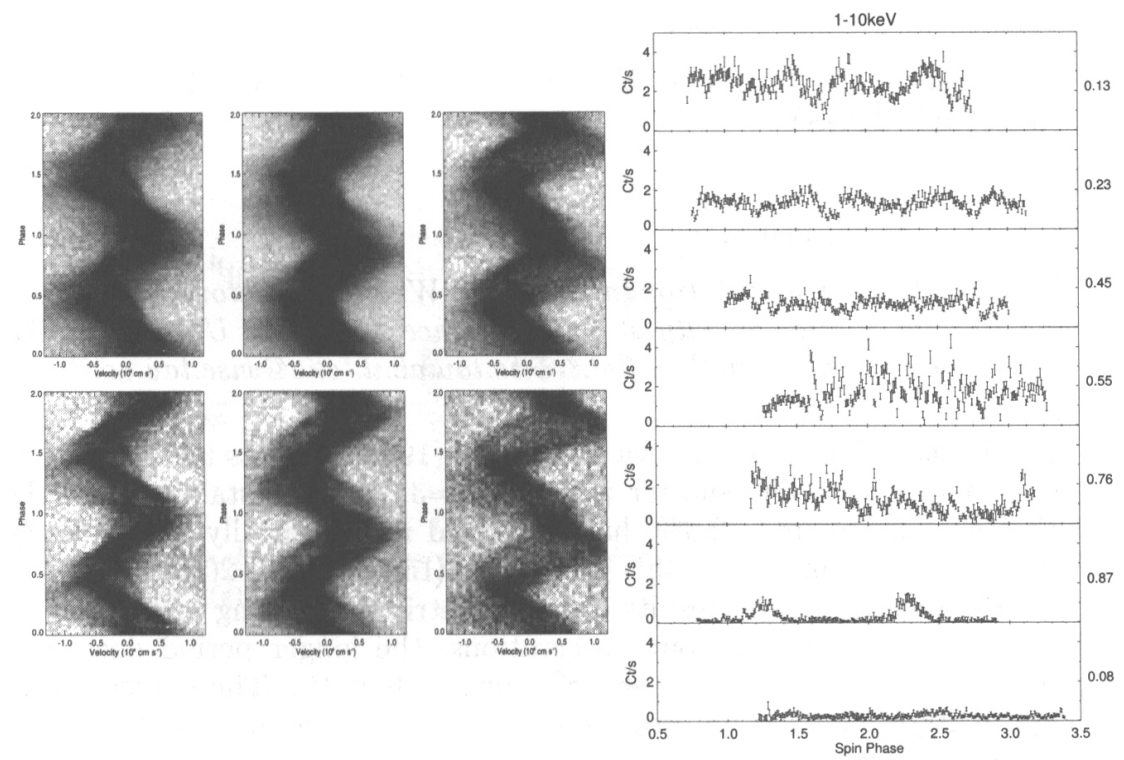

Figure 1. Left: Phase folded line profiles of $\mathrm{H} \beta$ (top) and HeII 4686 (bottom) on each of the three nights (from left to right) July 20, 22 and 24. Right: XMM-Newton light curves in the $1-10 \mathrm{keV}$ band folded on the orbital period for successive nights over the beat cycle. The beat cycle phases (arbitrary zero point) are shown on the right of each plot.

RXTE X-ray light curves (Ramsay et al 2000). Accretion is mainly at one pole in the lower hemisphere at beat phase 0.87 , while it is clearly also accreting at a pole in the upper hemisphere at most other phases (for example phase 0.23 ). The X-ray spectra also change appreciably at different beat phases: one (at beat phase 0.13 ) requires a distinct soft X-ray component while the other (at phase 0.55 ) does not. Similar behaviour is seen in our shorter XMM XMM-Newton observations of the asynchronous polar BY Cam (Ramsay \& Cropper 2002). A comprehensive analysis is in progress.

\section{References}

Ramsay, G., \& Cropper, M. 2002, MNRAS, 336, 1129

Ramsay, G., Buckley, D. A. H., Cropper, M., \& Harrop-Allin, M.K. 1999, MNRAS, 303, 96

Ramsay, G., Potter, S., Cropper, M., Buckley, D.A.H., \& Harrop-Allin, M.K. 2000, MNRAS, 316, 225

Schwope, A., Buckley, D.A.H., O’Donoghue, D., Hasinger, G., Trümper, J., \& Voges, W. 1997, A\&A, 326, 195 